\title{
EARLY ULTRAVIOLET/OPTICAL EMISSION OF THE TYPE Ib SN 2008D
}

\author{
Melina C. Bersten ${ }^{1}$, Masaomi Tanaka ${ }^{2}$, Nozomu Tominaga ${ }^{3}$, Omar G. Benvenuto ${ }^{4,5}$, and Ken’ichi Nomoto ${ }^{1}$ \\ ${ }^{1}$ Kavli Institute for the Physics and Mathematics of the Universe, Todai Institutes for Advanced Study, University of Tokyo, \\ 5-1-5 Kashiwanoha, Kashiwa, Chiba 277-8583, Japan; melina.bersten@ipmu.jp \\ ${ }^{2}$ National Astronomical Observatory of Japan, 2-21-1 Osawa, Mitaka, Tokyo 181-8588, Japan \\ ${ }^{3}$ Department of Physics, Faculty of Science and Engineering, Konan University, 8-9-1 Okamoto, Kobe, Hyogo 658-8501, Japan \\ ${ }^{4}$ Facultad de Ciencias Astronómicas y Geofísicas, Universidad Nacional de La Plata, Paseo del Bosque S/N, B1900FWA La Plata, Argentina \\ Received 2012 October 25; accepted 2013 February 26; published 2013 April 5
}

\begin{abstract}
We propose an alternative explanation for the post-breakout emission of SN 2008D associated with the X-ray transient 080109. Observations of this object show a very small contrast of 0.35 dex between the light-curve minimum occurring soon after the breakout, and the main luminosity peak which is due to radioactive heating of the ejecta. Hydrodynamical models show that the cooling of a shocked Wolf-Rayet star leads to a much greater difference ( $\gtrsim 0.9$ dex). Our proposed scenario is that of a jet produced during the explosion which deposits ${ }^{56} \mathrm{Ni}-\mathrm{rich}$ material in the outer layers of the ejecta. The presence of high-velocity radioactive material allows us to reproduce the complete luminosity evolution of the object. Without outer ${ }^{56} \mathrm{Ni}$ it could be possible to reproduce the early emission purely from cooling of the shocked envelope by assuming a larger progenitor than a Wolf-Rayet star, but that would require an initial density structure significantly different from what is predicted by stellar evolution models. Analytic models of the cooling phase have been proposed reproduce the early emission of SN 2008D with an extended progenitor. However, we found that the models are valid only until 1.5 days after the explosion where only two data of SN 2008D are available. We also discuss the possibility of the interaction of the ejecta with a binary companion, based on published analytic expressions. However, the binary separation required to fit the early emission should be $\lesssim 3 R_{\odot}$, which is too small for a system containing two massive stars.
\end{abstract}

Key words: hydrodynamics - supernovae: general - supernovae: individual (SN 2008D)

Online-only material: color figures

\section{INTRODUCTION}

Supernova (SN) 2008D attracted a good deal of attention because of its unusual observational characteristics. Most outstandingly, the serendipitous detection of the X-ray transient (XRT) 080109 associated with the SN explosion during the Swift follow-up of SN 2007uy, of another SN in the same galaxy, NGC 2770 (Berger \& Soderberg 2008; Kong \& Maccarone 2008). The optical counterpart of the XRT was revealed at the same position of the transient a few hours after, providing unprecedented early coverage of the SN emission. Initially the SN was classified as a broad-line Type Ic SN (SN Ic; Blondin et al. 2008; Valenti et al. 2008) based on the broad absorptions and lack of hydrogen and helium in the first spectrum. Later on, however, the spectra revealed the presence of strong He I lines, which changed the classification to Type Ib (Modjaz et al. 2008; Malesani et al. 2009).

Type Ib SNe, as well as Type Ic and the transitional Type IIb $\mathrm{SNe}$, are believed to be the result of the core collapse of massive stars $\left(M_{\text {ZAMS }} \gtrsim 10 M_{\odot}\right)$ that have lost most or all of their hydrogen (and often helium) layers before the explosion. For that reason they are called "stripped-envelope SNe" (Clocchiatti et al. 1996). However, the mechanism by which the envelope is removed is not fully understood. Strong winds of single massive progenitors $\left(M \gtrsim 30 M_{\odot}\right)$, sudden eruptions, and binary interaction have been proposed as possible explanations (Maeder \& Meynet 1994; Nomoto et al. 1995; Owocki et al. 2004 among others), with the binary origin being the most probable scenario. In all cases a Wolf-Rayet (WR) structure

\footnotetext{
5 O.G.B. is member of the Carrera del Investigador Científico de la Comisión de Investigaciones Científicas de la Provincia de Buenos Aires (CIC), Argentina.
}

is expected before the explosion, although for $\mathrm{SNe} \mathrm{IIb}$ a thin $\mathrm{H}$ envelope is expected to remain before the explosion (e.g., see SN, 1993J, Nomoto et al. 1993; Podsiadlowski et al. 1993; and SN 2011dh, Bersten et al. 2012). Interest in these objects has recently grown due to their connection with long gammaray bursts (GRBs). To date six $\mathrm{SNe}$ have been associated with GRB, one of which also showed XRT. In all of these cases a highly energetic Type Ic SN, also called "hypernova" was observed. Note, however, that the term hypernova is more generally used and refers to $\mathrm{SNe}$ with high luminosity or unusually broad lines, independently of their association with GRB. The origin of hypernovae is thought to be a rapidly rotating and accreting compact object (MacFadyen et al. 2001), or a magnetar (Thompson et al. 2004), which should produce relativistic outflows.

The nature of the XRT associated with SN 2008D is controversial. Some authors are in favor of the supernova shock-breakout origin (Soderberg et al. 2008; Chevalier \& Fransson 2008), while others consider that the transient was caused by a mildly relativistic jet penetrating through the envelope of the progenitor star (Mazzali et al. 2008; Li 2008; $\mathrm{Xu}$ et al. 2008). In the latter case, the XRT could represent a transition between the most energetic hypernovae and standard core-collapse SNe. However, the presence of a GRB or the radio detection of superluminal motions caused by a longlived relativistic outflow were firmly ruled out for SN 2008D (Soderberg et al. 2008; Bietenholz et al. 2009). Nevertheless, the lack of confirmation of a thermal component for the XRT (Modjaz et al. 2009), and the strong evidence of an asymmetric explosion (Modjaz et al. 2009; Gorosabel et al. 2010), possibly bipolar (Tanaka et al. 2009b; Maund et al. 2009), leaves room for the mildly relativistic-jet scenario. 
Another interesting feature of SN 2008D was its doublepeaked optical light curves (LCs). The first peak occurred at $\approx 1$ day after the XRT, and was similar to that of SN 2006aj, which was connected with a GRB (Campana et al. 2006). The second and main peak happened at $\approx 20$ days after the XRT, consistently with other $\mathrm{SNe} \mathrm{Ib}$ and Ic, and its origin is related with the decay of radioactive material synthesized during the explosion. By modeling the emission around the main peak, explosion parameters of SN 2008D, such as ejecta mass $\left(M_{\mathrm{ej}}\right)$, kinetic energy $\left(E_{K}\right)$, and nickel mass $\left(M\left({ }^{56} \mathrm{Ni}\right)\right)$, were estimated in previous studies. Using analytic models, Soderberg et al. (2008) found $M_{\mathrm{ej}}=3-5 M_{\odot}$, and $E_{K}=2-4$ foe (1 foe $\left.=1 \times 10^{51} \mathrm{erg} \mathrm{s}^{-1}\right)$. Based on Monte Carlo simulations, Mazzali et al. (2008) suggested that $M_{\mathrm{ej}}=7 M_{\odot}, E_{K}=6$ foe, and $M\left({ }^{56} \mathrm{Ni}\right)=0.09 M_{\odot}$. Tanaka et al. $(2009 \mathrm{a}$, T09 hereafter) subsequently presented an exhaustive analysis of SN 2008D using a set of progenitor models and hydrodynamics/ nucleosynthesis calculations. They found a very good agreement with the LC and the spectra for a model with $M_{\mathrm{ej}}=5.3 \pm 1 M_{\odot}$, $E_{K}=6 \pm 2.5$ foe, and $M\left({ }^{56} \mathrm{Ni}\right)=0.07 M_{\odot}$.

Early UV/optical emission is expected to occur after the arrival of the shock wave at the surface of the progenitor (shock break-out) and before the re-brightening due to the decay of radioactive material. This emission is a consequence of the nearly adiabatic cooling due to the expansion of the outermost layers of the ejecta. The observations of the shock break-out and the following emission provide very valuable information about the structure of the star prior to the explosion. The duration of this early phase depends strongly on the size of the progenitor. For compact WR stars, which are proposed progenitors of SNe $\mathrm{Ib}$ and Ic, a duration of a few days is expected. Consequently, catching a SN during this phase is quite a challenge and only for a handful of objects has this been possible.

Naturally, the origin of the earliest part of the LC of SN 2008D has been associated in the literature with the adiabatic cooling of the outer layers of the ejecta (Soderberg et al. 2008; Modjaz et al. 2009; Rabinak \& Waxman 2011). However, different authors arrived at different conclusions regarding the progenitor radius. Some suggested a value of $\approx 1 R_{\odot}$ (Soderberg et al. 2008; Rabinak \& Waxman 2011) while others proposed a larger value of $\approx 9 R_{\odot}$ (Modjaz et al. 2009; Chevalier \& Fransson 2008). In all these cases the estimations were based on analytic models for the early emission. Note that Mazzali et al. (2008) and T09 performed numerical simulations, but they did not attempt a hydrodynamical modeling of the early emission so as to help discriminate between both possibilities.

Given the unique information about the progenitor structure that is provided by the early emission, we have performed new hydrodynamical calculations for SN 2008D, focusing on this phase. Surprisingly, we found that our hydrodynamical models are not consistent with the cooling-phase explanation given in the literature for this object. Hydrodynamical models predict a much larger contrast between the luminosity minimum which occurs after the breakout, and the luminosity peak due to radioactive decay than what was observed for SN 2008D. This has also been noted recently by Dessart et al. (2011) using a set of hydrodynamical and spectral calculations of SNe Ib and Ic.

Here we present the first radiation-hydrodynamical models for the early emission of SN 2008D and propose an alternative explanation for this emission based on a double-peaked ${ }^{56} \mathrm{Ni}$ distribution. The data and hydrodynamic code employed are described in Section 2. A comparison with previous hydrodynamical results is shown in Section 3.1. Our proposed model for
SN 2008D is presented in Section 3.2. In Section 4 we compare our model with the analytic models used in the literature, and discuss alternative explanations for the early emission. Finally, our conclusions are presented in Section 5.

\section{DATA AND MODEL}

\subsection{Observational Material}

The bolometric LC (Lbol) of SN 2008D was calculated by Modjaz et al. (2009) using $U V W 1 B V R r^{\prime} I i^{\prime} J H K_{s}$ broadband photometry for $t<31$ days, referred to the onset of the XRT, i.e., $\mathrm{JD}=2454475.06$ (in what follows, all times will be referred to this moment). Here we adopt the estimates of Lbol from blackbody fits to the broadband photometry, which, according to Modjaz et al. (2009), are more accurate at early times than direct integration of the observed flux. At later times we include bolometric calculations provided by T09 (see their Appendix A) using optical and near-infrared (NIR) data obtained with the MAGNUM telescope and the Himalayan Chandra telescope. Also available in the literature are two earlier data points, one at 0.14 days observed with the Swift telescope in the UVW2, UVW1 and $U$ filters (Soderberg et al. 2008), and another at 0.44 days in the BVRI bands (Mazzali et al. 2008). The integrated flux for each of these observations was calculated, respectively, by the authors. Here we adopt, for $t=0.44$ days, the sum of the luminosity in the $B V R I$ bands plus the contribution of the UV as estimated from the earlier Swift observations. The uncertainty of this point was assumed to be the sum in quadrature of the uncertainties of both contributions. We denote this earliest point with a different symbol (square) in all figures where it is included in order to indicate its different origin. The distance and total reddening assumed in the calculations are $d=32 \mathrm{Mpc}$, and $E(B-V)=0.65 \mathrm{mag}$ (Mazzali et al. 2008).

The bolometric LC reveals a double-peaked shape that is also observed in the broad-band photometry (Soderberg et al. 2008; Mazzali et al. 2008). We assume throughout this paper that the earliest data point is confident and therefore the early peak shape of the LC is robust. The first peak occurred at about 1 day with a luminosity of $L=1 . \times 10^{42} \mathrm{erg} \mathrm{s}^{-1}$, and the second or main maximum happened at $\approx 19$ days with a luminosity of $L=1.62 \times 10^{42} \mathrm{erg} \mathrm{s}^{-1}$, corresponding to $M_{\mathrm{bol}}=-16.8 \mathrm{mag}$. Therefore, SN 2008D had a normal peak luminosity and a rise time at the long end of the observed range for $\mathrm{SNe} \mathrm{Ib}$ and Ic (Richardson et al. 2006; Drout et al. 2012). The contrast of luminosity between the main peak and the dip that occurs before the re-brightening of the LC due to radioactive material is only of 0.35 dex.

Apart from the bolometric LC, photospheric velocities and color temperatures are also compared with our models. We employ He-line and photospheric velocities derived from the spectral modeling by T09 and color temperatures calculated by Modjaz et al. (2009).

\subsection{Light Curve Models}

Synthetic LCs were calculated using the spherical, Lagrangian, LTE, hydrodynamical code described by Bersten et al. (2011, BBH11 hereafter). The code solves radiation transport in the flux-limited diffusion approximation, including $\gamma$-ray transfer in gray approximation. Any distribution of the radioactive material is allowed and the energy deposition due to radioactive decay is computed in each layer of the entire ejecta. The ionization structure is determined by solving the Saha equation taking 
into account the most relevant elements in the progenitor structure. The Rosseland mean opacity is calculated using OPAL tables (Iglesias \& Rogers 1996 and references therein), and the empirical relation used in T09, which is based on electron scattering opacity as derived from the TOPS database (Magee et al. 1995; Deng et al. 2005), and including effects of lines. The explosion is simulated by injecting near the center of the object a certain amount of energy in a thermal form during a short interval. The code does not explicitly solve for the explosive nucleosynthesis produced during the shock propagation, but it implicitly takes this into consideration in the chemical composition assumed for our initial models.

As pre-supernovae models, stellar evolution calculations of He stars by Nomoto \& Hashimoto (1988) were adopted. T09 studied five different He star models with masses of 4, 6, 8, 10 , and $16 M_{\odot}$ using a variety of explosion parameters (see their Table 1). They found that the models with a He mass of 6 and $8 M_{\odot}$ (He6 and He8, respectively) were most consistent with the spectra and LCs of SN 2008D. They derived a kinetic energy of $E_{K}=(6.0 \pm 2.5) \times 10^{51} \mathrm{erg}$, an ejecta mass of $M_{\mathrm{ej}}=5.3 \pm 1.0 M_{\odot}$, a ${ }^{56} \mathrm{Ni}$ mass of $M\left({ }^{56} \mathrm{Ni}\right)=0.07 M_{\odot}$, and a compact-remnant mass in the range of 1.6-1.8 $\mathrm{M}_{\odot}$.

We have based the current analysis on the results of T09, and so we calculated the hydrodynamics for these two optimal models (He6 and He8) which correspond to main-sequence masses of 20 and $25 M_{\odot}$, as derived from the $M_{\mathrm{MS}}-M_{\alpha}$ relation of Sugimoto \& Nomoto (1980). The chemical abundance distribution left by the explosive nucleosynthesis is assumed to be a pre-explosion condition (see T09 for more details). One characteristic of these nucleosynthesis calculations in spherical symmetry is that ${ }^{56} \mathrm{Ni}$ is confined in the innermost layers of the ejecta, which makes it very difficult to explain the timescale of the rising part of the LC normally observed for $\mathrm{SNe} \mathrm{Ib}$ and Ic. Instead, a more extensive mixing of ${ }^{56} \mathrm{Ni}$, associated with multi-dimensional effects, was used by T09 and we also adopted it here (see Section 3.1 for more details). Note that mixing ${ }^{56} \mathrm{Ni}$ with a large radius of the ejecta of CCSNe has been successfully produced in recent three-dimensional numerical calculations (Hammer et al. 2010; Joggerst et al. 2010). In addition, Dessart et al. (2012) have claimed that all SNe classified as Ib require efficient mixing of ${ }^{56} \mathrm{Ni}$ in the helium-rich layers.

\section{HYDRODYNAMIC MODEL OF SN 2008D}

We present here our hydrodynamic calculations for SN 2008D using the He6 and He8 initial models. In Section 3.1 we compare our results with those of T09 by focusing on the second peak of the bolometric LC ( $t>5$ days), which determines the global properties of the SN. The early emission is analyzed in Section 3.2, where we present a model that consistently reproduces the first and second peaks of the LC.

\subsection{Comparison with T09}

As is well known, the shape of the LC depends on the explosion kinetic energy $E_{K}$, the ejected mass $M_{\mathrm{ej}}$, and the mass and distribution of ${ }^{56} \mathrm{Ni}$. Analytic expressions for these dependencies were given by Arnett (1982), where the width of the LC peak is $\tau_{\mathrm{LC}} \propto M_{\mathrm{e} j}^{3 / 4} E_{K}^{-1 / 4}$, and the peak luminosity is $L_{\text {peak }} \propto M_{\mathrm{Ni}} \tau_{\mathrm{LC}}^{-1}$. Therefore, it is possible to estimate explosion parameters by comparing models and observed bolometric LCs. However, various combinations of $M_{\mathrm{ej}}$ and $E_{K}$ can fit the LC, and spectra modeling is needed in order to break the degeneracy of the parameters. This type of analysis was done by T09 and

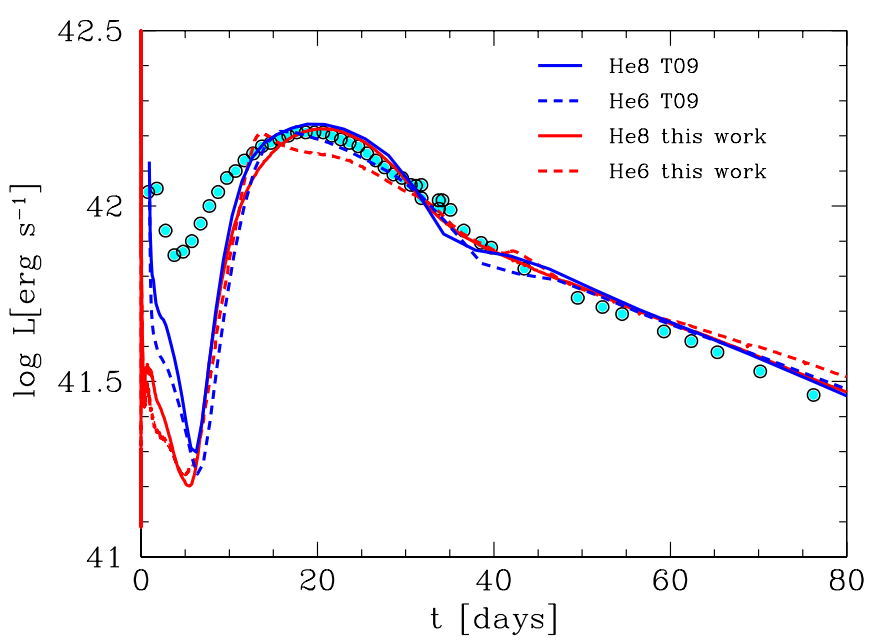

Figure 1. Comparison between the bolometric LC for models He6 (blue lines) and He8 (red lines) calculated in this work (solid lines) and by T09 (dashed lines). Both works adopted the same progenitor models and physical parameters. The differences are only related with the hydrodynamic code used (see Section 3.1 for more details). The observed bolometric LC of SN 2008D is also included (cyan dots).

(A color version of this figure is available in the online journal.)

those authors found a very good agreement between models and observations using the He6 and He8 initial models with the following explosion parameters: for $\mathrm{He} 6, E_{K}=3.7$ foe, a cut mass of $M_{\text {cut }}=1.6 M_{\odot}$, and $M_{\mathrm{Ni}}=0.065 M_{\odot}$; and for $\mathrm{He} 8$, $E_{K}=8.4$ foe, $M_{\text {cut }}=1.8 M_{\odot}$, and $M_{\mathrm{Ni}}=0.07 M_{\odot}$. A constant ${ }^{56} \mathrm{Ni}$ distribution up to $7000(9000) \mathrm{km} \mathrm{s}^{-1}$ for model He6 (He8) was assumed to account for the rise time to the main peak.

We have used the code of BBH11 to calculate bolometric LCs and photospheric evolution for the same models (He6 and He8) and explosion parameters as those of T09. As opposed to T09, our calculations solve the hydrodynamics coupled to the radiative transfer, allowing us to model consistently the earliest phases of the $\mathrm{SN}$ evolution. Figure 1 shows a comparison of our results with those of T09, along with the observed bolometric LC of SN 2008D. The photospheric velocities are compared in Figure 2 where we include the He lines velocities as well as the photospheric velocity estimated from spectra modeling by T09. From these figures we see that the agreement between both models is reasonable, considering the differences in the calculation methods. The LCs look remarkably similar for times $t \gtrsim 5$ days. It is not striking that the largest differences appear at the earliest epochs since the code in BBH11 self-consistently calculates the shock wave propagation, the breakout, and the later evolution, while in the calculations of T09 the hydrodynamics and the radiative transport were computed with different codes, switching from one code to another when the homologous expansion was achieved. There is a small systematic difference between the photospheric velocities of both models. This is probably related to slight differences in the re-gridding of the initial model, which produces a small difference in the actual total mass of the progenitor (of $\left.\approx 0.4 M_{\odot}\right)$.

Both our calculations and those of T09 provide very good matches to the observations around the main peak. Note that model He8 is the one that best represents the bolometric LC, while model He6 gives a better fit to the velocities, especially the photospheric velocities. Some intermediate model between these two seems to be the most plausible progenitor, as suggested in T09. However, at times before eight days, the models predict 


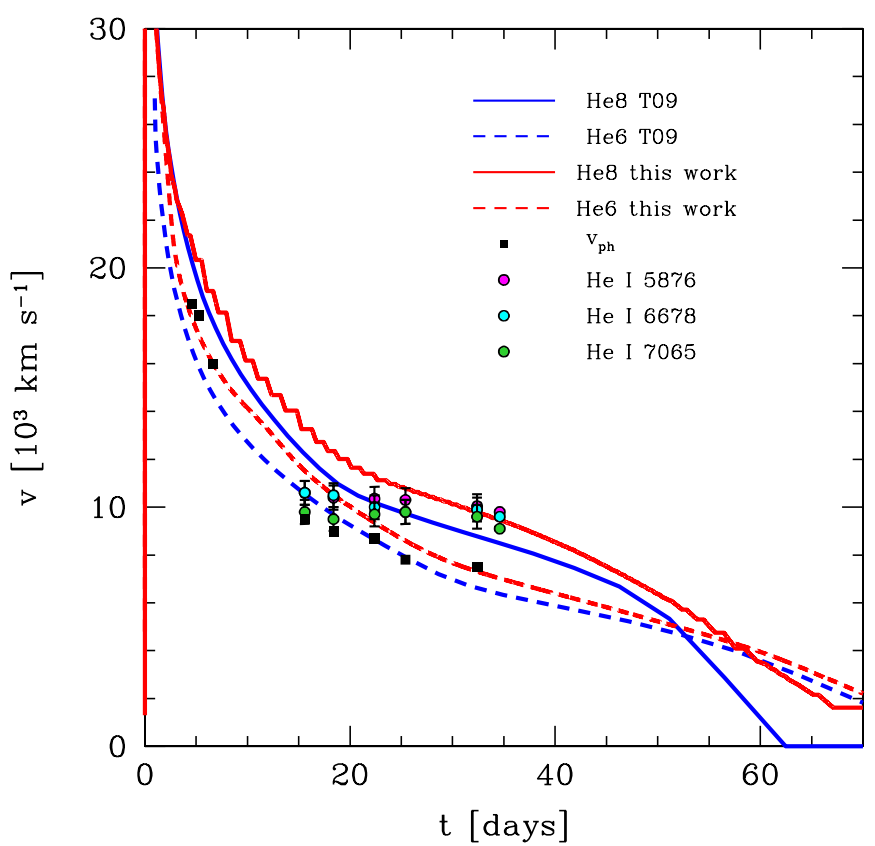

Figure 2. Evolution of the photospheric velocity for models He6 (blue lines) and He8 (red lines) calculated in this paper (solid lines) and by T09 (dashed lines). For comparison, observed He line velocities (circles) and photospheric velocities estimated through spectra modeling by T09 are also shown.

(A color version of this figure is available in the online journal.)

much lower luminosity than the observed one. As described in Section 3.2, this can be overcome by including a small amount of ${ }^{56} \mathrm{Ni}$ in the outermost layers of the ejecta.

\subsection{A High-velocity Blob of ${ }^{56} \mathrm{Ni}$}

It is clear from Figure 1 that the models presented in Section 3.1 cannot explain the first peak shown by the observations. The difference in luminosity between the hydrodynamical models and the observations at early times are larger than 0.5 dex, which is much greater than any possible uncertainty related to the calculations and observations. The models predict a brightness contrast between the dip and the main peak that is larger than 0.9 dex, while the observations show a contrast of only 0.35 dex. Although the early behavior of SN 2008D was attributed in the literature to the cooling of the outer envelope of the ejecta after being heated by the shock wave, our calculation suggests that this cooling happened very quickly, and in less than one day most of the energy deposited by the shock was degraded. Therefore, unless we assume a different structure for the progenitor-i.e., lager radius and/or more massive external envelope-some source of additional energy in the outer layers of the ejecta is required in order to reproduce the first peak of the LC. Here we study this possibility and leave the discussion of other alternatives for Section 4.

We artificially placed some ${ }^{56} \mathrm{Ni}$ in the outer layers of the ejecta as the source of extra energy needed to explain the first peak. This material may have been carried by a jet-like phenomenon produced during an aspherical explosion. The presence of a jet was proposed by Mazzali et al. (2008) to explain the XRT associated with SN 2008D, as well as the broad-line appearance of the spectrum at $t \lesssim 3-5$ days. The double-peaked oxygen lines detected in late spectra (Malesani et al. 2009; Modjaz et al. 2009; Tanaka et al. 2009b) provide additional evidence of the asphericity during the explosion. A

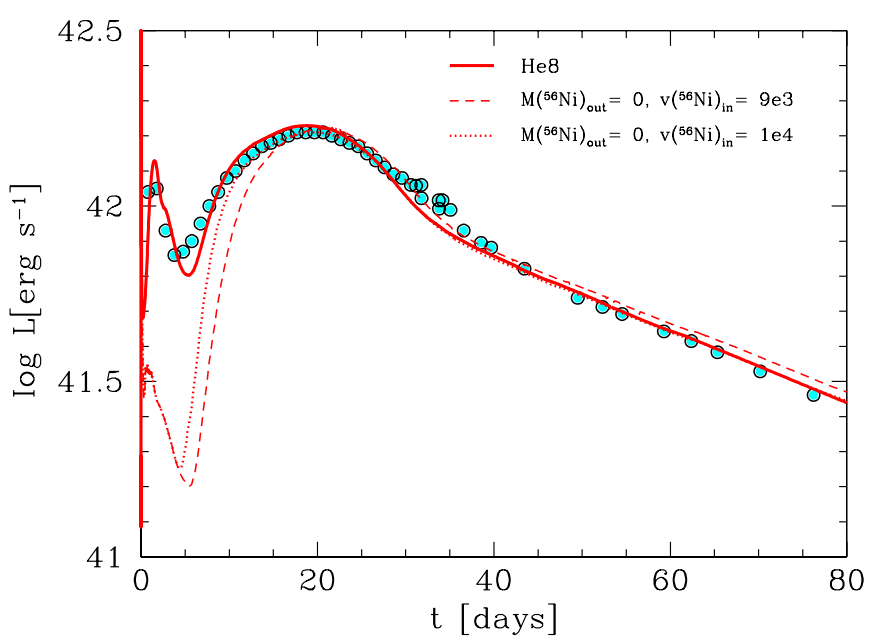

Figure 3. Bolometric LC of our optimal model (solid line) compared with the observations of SN 2008D. This model can consistently reproduce the two observed peaks. For comparison, two models without external ${ }^{56} \mathrm{Ni}$ and slightly different internal ${ }^{56} \mathrm{Ni}$ distributions are shown. A model with ${ }^{56} \mathrm{Ni}$ mixed up to $9000 \mathrm{~km} \mathrm{~s}^{-1}$ as assumed in T09 is shown by a dashed line, and a model with the same internal ${ }^{56} \mathrm{Ni}$ distribution used in the optimal model, i.e., up to $\approx 10000 \mathrm{~km} \mathrm{~s}^{-1}$ is shown by a dotted line. Note that both models without the external ${ }^{56} \mathrm{Ni}$ show a difference with the observations larger than $0.5 \mathrm{dex}$ at early times.

(A color version of this figure is available in the online journal.)

spectropolarimetry study of SN 2008D by Maund et al. (2009) suggest that a jet was produced but that it stalled in the $\mathrm{C}+\mathrm{O}$ core. Maund et al. base this conclusion mainly on the observed low degree of $\mathrm{O}_{\mathrm{I}}$ line polarization. However, for this $\mathrm{SN}$ the O I $\lambda 7774$ line is so weak that one cannot expect any strong polarization associated with it. Weak lines do not produce high polarization, as shown, for example, in Tanaka et al. (2012).

A double-peaked ${ }^{56} \mathrm{Ni}$ distribution was previously suggested to model the double-peaked light curve of SN 2005bf (Tominaga et al. 2005; Folatelli et al. 2006). The presence of an unobserved jet in SN 2005bf was speculated as being responsible for the anomalous ${ }^{56} \mathrm{Ni}$ distribution. Despite the overall observational differences between SN 2005bf and SN 2008D, some similarities in the polarization properties of both objects were found (Maund et al. 2009) that could indicate similarities in the explosion geometry.

In Figure 3 we show the bolometric LC for our model with ${ }^{56} \mathrm{Ni}$ in the outer layers (solid line) compared with the observations. This "optimal model" is similar to the He8 model presented in Section 3.1 (dashed line in the figure) but with an extra amount of $0.01 M_{\odot}$ of ${ }^{56} \mathrm{Ni}$ in the outermost layers of the ejecta (at $v>20,000 \mathrm{~km} \mathrm{~s}^{-1}$ ) added to explain the early emission at $t \lesssim 5$ days. In addition, the optimal model has a slightly extended distribution of internal ${ }^{56} \mathrm{Ni}$ up to $\approx 10000 \mathrm{~km} \mathrm{~s}^{-1}$ (as compared with $9000 \mathrm{~km} \mathrm{~s}^{-1}$ that was used in the model of T09) to improve the agreement with the data during the rise to the main peak. For clarity, in Figure 3 we show two models that lack external ${ }^{56} \mathrm{Ni}$, with internal distributions up to 9000 and $10000 \mathrm{~km} \mathrm{~s}^{-1}$ (dashed and dotted lines, respectively). In the rest of this paper the model without external ${ }^{56} \mathrm{Ni}$ will be the latter one and will be referred to as He8.

The agreement between the optimal model and the observations is excellent. The assumption of external ${ }^{56} \mathrm{Ni}$ allowed us to reproduce the first and second peaks consistently. The luminosity contrast between dip and main peak is now similar to that shown by the observations. 


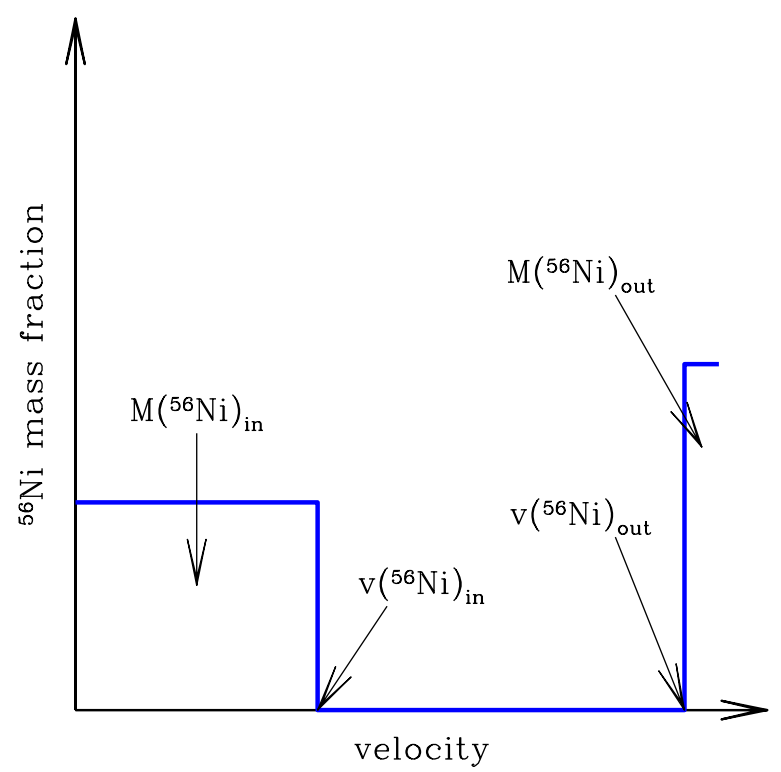

Figure 4. Schematic doubly-peaked ${ }^{56} \mathrm{Ni}$ distribution characterized by the following parameters: the mass of internal ${ }^{56} \mathrm{Ni}, M\left({ }^{56} \mathrm{Ni}\right)_{\text {in }}$, the mass of external ${ }^{56} \mathrm{Ni}, M\left({ }^{56} \mathrm{Ni}\right)_{\text {out }}$, the minimum velocity for the external ${ }^{56} \mathrm{Ni}, v\left({ }^{56} \mathrm{Ni}\right)_{\text {out }}$ and the maximum velocity for the internal ${ }^{56} \mathrm{Ni}, v\left({ }^{56} \mathrm{Ni}\right)_{\text {in }}$.

(A color version of this figure is available in the online journal.)

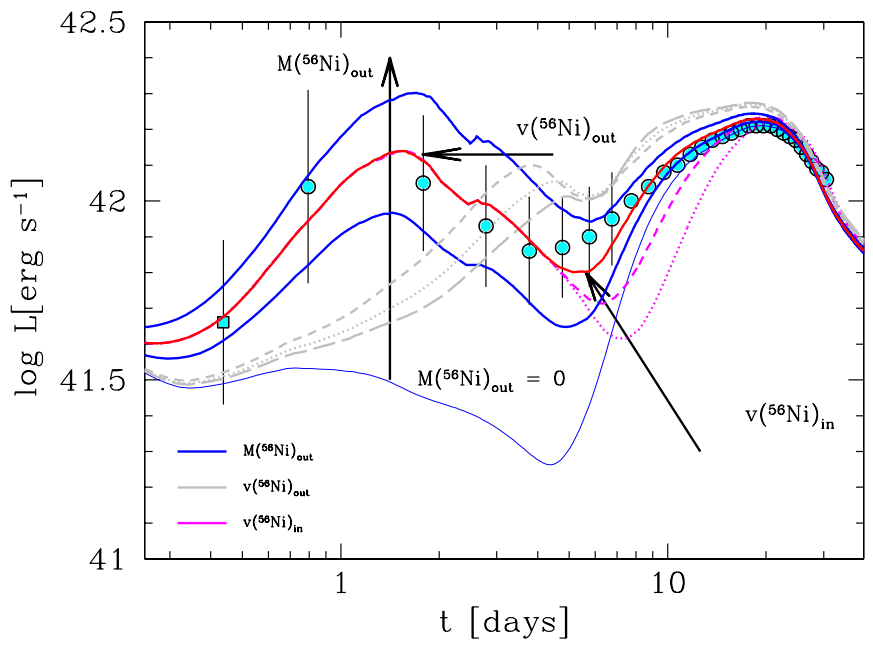

Figure 5. Sensitivity of the early LC on the distribution of ${ }^{56} \mathrm{Ni}$. Three different values of $M\left({ }^{56} \mathrm{Ni}\right)_{\text {out }}, v\left({ }^{56} \mathrm{Ni}\right)_{\text {out }}$ and $v\left({ }^{56} \mathrm{Ni}\right)_{\text {in }}$ have been used (see Figure 4). The arrows indicate the change produced in the LC by the increase of each parameter. A model without ${ }^{56} \mathrm{Ni}$ is shown for comparison. Time is plotted in logarithmic scale to show more clearly the behavior of the early LC.

(A color version of this figure is available in the online journal.)

In order to obtain our optimal model, several ${ }^{56} \mathrm{Ni}$ distributions were explored. In Figure 4 we schematically show a double-peaked ${ }^{56} \mathrm{Ni}$ distribution where the internal and external components were assumed to be step-like functions within some internal and external velocities. This distribution can be characterized with three parameters: the mass of external ${ }^{56} \mathrm{Ni}, M\left({ }^{56} \mathrm{Ni}\right)_{\text {out }}$, the minimum velocity for the external ${ }^{56} \mathrm{Ni}$, $v\left({ }^{56} \mathrm{Ni}\right)_{\text {out }}$, and the maximum velocity for the internal ${ }^{56} \mathrm{Ni}$, $v\left({ }^{56} \mathrm{Ni}\right)_{\text {in }}$. The effect of the variation of these parameters on the early LC is shown in Figure 5. We see that (1) larger $M\left({ }^{56} \mathrm{Ni}\right)_{\text {out }}$ produces a more luminous first peak, (2) higher $v\left({ }^{56} \mathrm{Ni}\right)_{\text {out }}$ produces an earlier first peak, and (3) lower $v\left({ }^{56} \mathrm{Ni}\right)_{\text {in }}$ translates to deeper and later minimum, or later rise to the second peak. Specifically, we have adopted the following values for our opti-

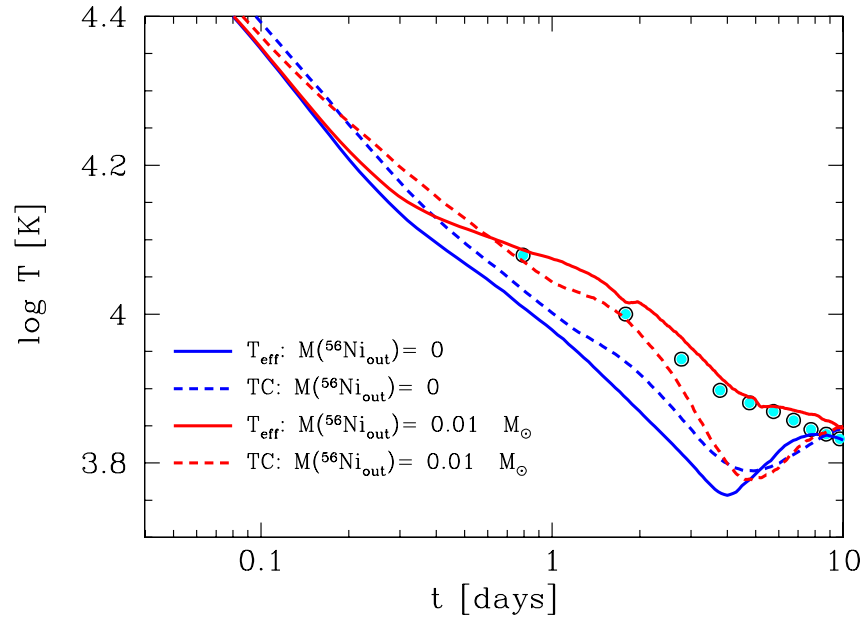

Figure 6. Color (dashed lines) and effective temperature (solid lines) evolution for our optimal model (red) and for the same model without external ${ }^{56} \mathrm{Ni}$ (blue). The color temperature of SN 2008D calculated by Modjaz et al. (2009) using broad-band photometry is shown for comparison (dots).

(A color version of this figure is available in the online journal.)

mal model: $M\left({ }^{56} \mathrm{Ni}\right)_{\text {out }}=0.01 M_{\odot}, v\left({ }^{56} \mathrm{Ni}\right)_{\text {out }}=22000 \mathrm{~km} \mathrm{~s}^{-1}$, and $v\left({ }^{56} \mathrm{Ni}\right)_{\text {in }}=10000 \mathrm{~km} \mathrm{~s}^{-1}$.

Although we do not provide a detailed calculation to account for the occurrence of such ${ }^{56} \mathrm{Ni}$ distribution, it is remarkable that it allows us to obtain a very good fit to the observation and also to explore its effect on the early LC. We also explored the possibility of using smoother functions for the ${ }^{56} \mathrm{Ni}$ distribution, but we found that they failed to reproduce the two peaks shown in the observations, and instead they tended to produce a plateau-like shape. Given the large uncertainties in the early observations, we emphasize that the critical feature of our model is to have some ${ }^{56} \mathrm{Ni}$ in the outer layers but not the exact amount and shape of its distribution.

Another important parameter to compare with the observations is temperature. Figure 6 shows the effective (solid lines) and color (dashed lines) temperatures for the optimal model (red) and the model without external ${ }^{56} \mathrm{Ni}$ (blue), compared with the observed color temperature of SN 2008D (Modjaz et al. 2009).

The color temperature $\left(T_{C}\right)$ gives information about the continuum spectral energy distribution. To calculate $T_{C}$ from the models one must know the layer in the ejecta where the spectrum is formed. An approximate estimation of $T_{C}$ is given by the temperature at the "thermalization" depth (Ensman \& Burrows 1992), below which the gas and radiation field are in equilibrium. Following Ensman \& Burrows (1992), we calculated the "thermalization" depth as the layer where $3 \tau_{\text {abs }} \tau_{\text {sct }} \approx 1$. Here $\tau_{\text {sct }}$ is the optical depth for scattering and $\tau_{\text {abs }}$ the optical depth for absorption determined using $\kappa_{\text {abs }}=\kappa-\kappa_{\text {sct }}$ where $\kappa$ is the Rosseland mean opacity calculated with OPAL tables and $\kappa_{\text {sct }}$ is the scattering opacity calculated by solving the Saha equations. The effective temperature is defined as $T_{\text {eff }}^{4}=L /\left(4 \pi \sigma R_{\mathrm{ph}}^{2}\right)$, where $R_{\mathrm{ph}}$ is the radial coordinate at the photosphere position defined as the layer where $\tau=1$ and $L$ is the radiative luminosity plus the luminosity of ${ }^{56} \mathrm{Ni}$ decay deposited above the photosphere. ${ }^{6}$ From Figure 6 we see, as

\footnotetext{
6 As stated in Section 2.2, we have solved the gamma-ray transfer which allows us to compute the actual deposition of energy of gamma-ray in the entire ejecta (in particular, above the photosphere). Such deposition can be appreciably lower than the total available radioactive energy release, especially when the envelope becomes very dilute.
} 
expected, that the model without external ${ }^{56} \mathrm{Ni}$ has a higher color than effective temperature, but neither provides a satisfactory agreement with the data. On the other hand, the temperatures of the optimal model compare acceptably well with the data, but unlike what is expected, the effective temperature is higher than the color temperature for $t \gtrsim 1$ day. This can be understood from our definition of the effective temperature, which includes the extra luminosity of ${ }^{56} \mathrm{Ni}$ decay above the photosphere.

Unlike luminosity and temperature, the photospheric velocity is almost unaffected by the existence of external ${ }^{56} \mathrm{Ni}$. Therefore, we do not present a comparison between the velocities of the optimal model and observations because this is essentially the same as we presented in Section 3.1 and Figure 2.

One could expect that the presence of ${ }^{56} \mathrm{Ni}$ and associated iron-group elements in the outer layers can introduce lines and increase the blanketing, thus affecting the observed spectrum. However, if the iron-rich material is confined to a small solid angle, as in the case of a jet, the effect will be diluted, as explained by Dessart et al. (2012).

As our simulations are carried out in one dimension, we do not have the ability of reproducing the actual structure of a jet-like distribution. Nevertheless, in the proposed scenario only a small fraction of the mass is involved in the jet itself, and thus does not affect the global explosion dynamics. The low level of continuum polarization found by Maund et al. (2009) indicates that departures from spherical symmetry should be small. This allows us to treat the problem approximately in spherical symmetry. Calculations performed in higher dimensions are required to produce a self-consistent model that would allow to test the proposed jet and aspherical ${ }^{56} \mathrm{Ni}$ distribution.

\section{ALTERNATIVE APPROACHES}

\subsection{Cooling of the Shocked Envelope}

In the literature, the early emission of the LC of SN 2008D was explained as a consequence of the cooling of the outer stellar envelope following the passage the shock through the star and its subsequent breakout. Analytic models by Waxman et al. (2007, W07) and Chevalier \& Fransson (2008, CF08) were used in Soderberg et al. (2008) and Modjaz et al. (2009) to compare with the early data of SN 2008D. These models describe the emission of the outer layer of the ejecta assuming (1) pre-explosion density $\rho \propto(1-r / R)^{n}$, which is valid while the photosphere is in the outer shock-accelerated part of the ejecta as long as the mass above of the photosphere is less than $0.1 M_{\odot},(2)$ self-similar solution once the supernova reach the state of free expansion to determine the post-breakout density and velocity, and (3) constant opacity. The luminosity was calculated differently in each model. While W07 did not take into account the radiative diffusion assuming a strictly adiabatic expansion, CF08 considered the motion of a diffusion wave through the ejecta. Therefore these models are valid after free expansion is achieved and until more or less the onset of recombination, when the photosphere begins to recede into the ejecta. Recently, Rabinak \& Waxman (2011, RW11) improved the model of W07 by including the effect of recombination on the opacity. They also corrected a typographical error found in a coefficient of the Equation (19) of W07.

The analytic models mentioned above provide expressions for the time evolution of the luminosity, photospheric radius and temperature as a function of ejecta mass $\left(M_{\mathrm{ej}}\right)$, ejecta kinetic energy $\left(E_{K}\right)$, and progenitor radius $(R)$, in addition to other parameters that depend on the structure of the progenitor.

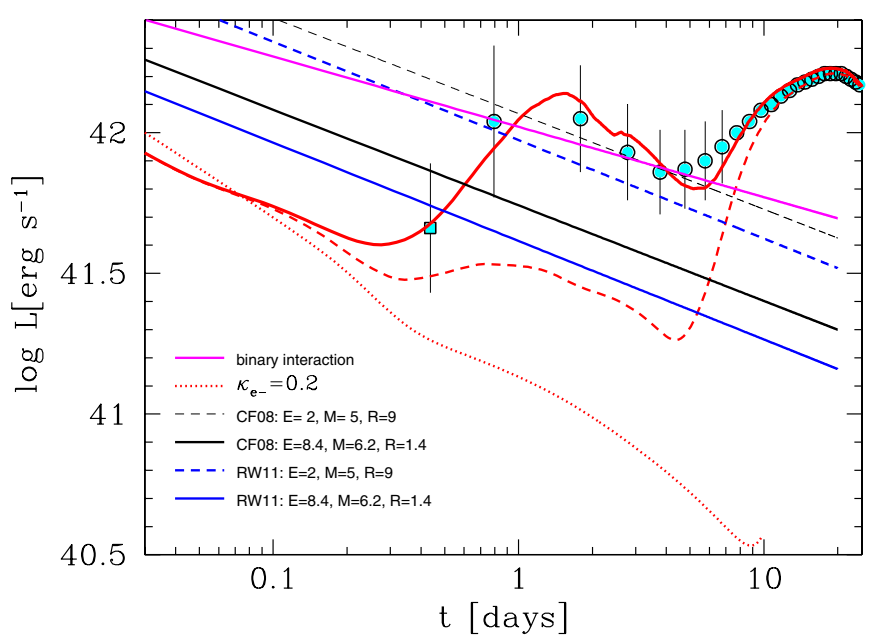

Figure 7. Comparison between the bolometric luminosity of the analytic and hydrodynamical models. The data are shown as cyan dots. Three different hydrodynamical calculations are shown: the optimal model (solid line), the same model but without external ${ }^{56} \mathrm{Ni}$ (dashed line), and a model assuming a constant opacity equal to the electron-scattering opacity for pure helium material, $\kappa_{e^{-}}=0.2 \mathrm{~g} \mathrm{~cm}^{-3}$, (dotted line). The analytic models of CF08 (black) and RW11 (blue) for the same explosion parameters as in our hydrodynamic simulations are also shown (solid line). The dashed lines show the same analytic models for an alternative set of physical parameters that include a larger initial radius. Note that a larger radius provides a better fit to the observations, with the exception of the earliest point. The analytic prediction of the binary interaction model of Kasen (2010) is also included (see Section 4.3 for additional information).

(A color version of this figure is available in the online journal.)

However, the dependence of luminosity and temperature on $R$ is stronger than on the other parameters. It is linear for the luminosity and $\propto R^{1 / 4}$ for the temperature.

In Figure 7 we show a comparison between our hydrodynamical calculations and the analytic predictions of CF08 and RW11. Three different hydrodynamical calculations are shown: the optimal model, the same model without external ${ }^{56} \mathrm{Ni}$, and a model with constant opacity equal to the electron-scattering opacity for pure helium, $\kappa_{e^{-}}=0.2 \mathrm{~g} \mathrm{~cm}^{-3}$. For the analytic models, the corrected expression for constant opacity from RW11 (their Equation (13)) was used here instead of Equation (19) of W07. Two sets of explosion parameters for models CF08 and RW11 are shown. First, we focus on the behavior of the analytic prediction with the same explosion parameters as in our hydrodynamic simulations (i.e., $E_{K}=8.4$ foe, $M_{\text {ej }}=6.2 M_{\odot}$, and $\left.R=1.4 R_{\odot}\right)$. The analytic models for compact stellar structures assume an initial density profile with index $n=3$, which is similar to the shape of the initial structure from stellar evolution that we adopted for model He8, as shown in the inset of Figure 8. As the proportionality constant between $\rho$ and $r$ is not given in the analytic models, we have scaled the density of these models to match the value of the He8 model at $0.1 M_{\odot}$ inside the star, i.e., where the analytic models are valid.

From Figure 7 it is clear that (1) the analytics models predict a higher luminosity than the hydrodynamic model without external ${ }^{56} \mathrm{Ni}$, but they are still not consistent with the observations, and (2) the slope of the analytic and hydro models are quite similar until $t \approx 0.5 \mathrm{~d}$, when helium starts to recombine and the luminosity of the hydro model enters a nearly plateau phase for approximately five days. After that time, the heating by ${ }^{56} \mathrm{Ni}$ produces a re-brightening of the LC. Note that the presence of a post-breakout plateau for WR progenitors of different radii and masses has recently been reported in simulations by 


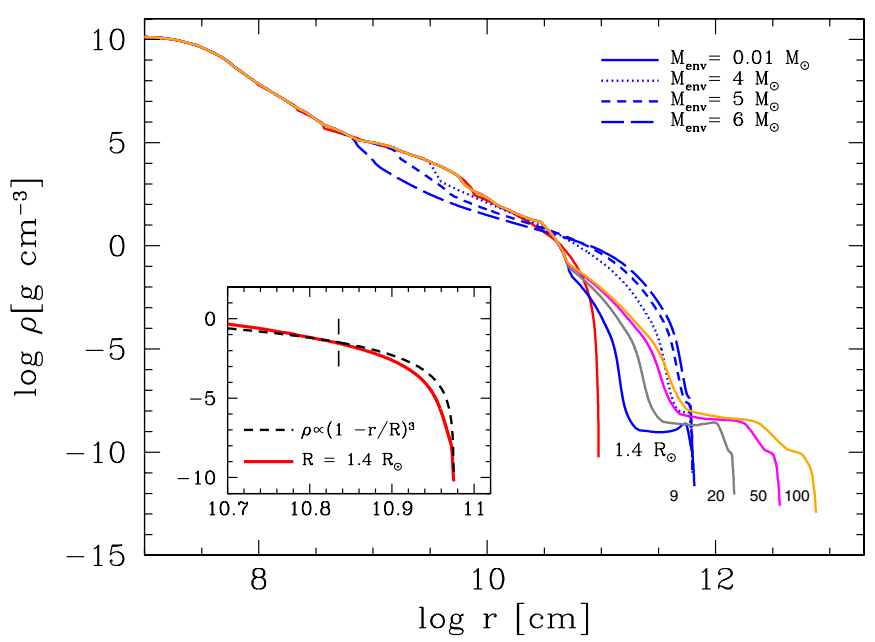

Figure 8. Initial density distributions as a function of radius for model $\mathrm{He} 8$ ( $R=1.4 R_{\odot}$; red line) and for models with different initial radii. Thick lines represent models whose variations in radius are accomplished by attaching essentially massless $\left(<0.01 M_{\odot}\right)$ envelopes to the He-rich layer of He8 model while thin lines show models with $9 R_{\odot}$ and massive envelopes attached at different points of mass inside the $\mathrm{He} 8$ model, as indicated in the upper right part of the figure. Inset: zoom of the outermost layers for model $\mathrm{He} 8$ ( $R=1.4 R_{\odot}$; red line) compared with the shape assumed for the analytic models: $\rho \propto(1-r / R)^{n}$, with $n=3$ and $R=1.4 R_{\odot}$ (dashed black line). The location of the limit of validity of the analytic models $\left(0.1 M_{\odot}\right.$ inside the star $)$ is indicated by vertical lines.

(A color version of this figure is available in the online journal.)

Dessart et al. (2011). In agreement with our simulations, they found that the typical differences between the luminosity of the post-breakout plateau $\left(1-5 \times 10^{7} L_{\odot}\right)$ and the main peak are much larger than the observed value of $\approx 0.3$ dex for SN 2008D.

Our hydrodynamical models show that the hypothesis of constant opacity breaks at $t \approx 0.5 \mathrm{~d}$ when He begins to recombine and the photosphere recedes into the ejecta. By $t \approx 1.5 \mathrm{~d}$, the mass above of the photosphere is larger than $0.1 M_{\odot}$ and the assumptions of the analytical models are no longer valid. Therefore, our simulations establish a limit of about 1.5 days for the validity of the analytic expressions. This is in close concordance with the range of validity suggested by Equations (16) and (17) of RW11, which for the values of $E_{K}, M_{\mathrm{ej}}$ and $R$ used here, give a range of up to two days. Note that for this range of time there are only two data points available, including the earliest point which does not seem to follow analytic predictions. This makes the conclusions derived from the analytic models more dubious.

Finally, as we noted before, there is a difference in luminosity between our models and the analytic models even for times earlier than 0.5 day. The differences may be related to the density structure of the outermost layers of the ejecta used in each calculation. A direct comparison of initial density structures is not possible because the scale of the relation between $\rho$ and $r$ is not provided in the analytic calculations. We can, however, make a quantitative comparison of post-shock breakout density structures. For the analytic models, this has the form $\rho \propto v^{n}$. Equation (1) of CF08 evaluated at $E=8.4$ foe, $M=6.4 M_{\odot}$, and $t=1.1$ days gives $\rho=7.9 \times$ $10^{3} v^{-10.18} \mathrm{~g} \mathrm{~cm}^{-3}$, where $v$ is expressed in $10^{8} \mathrm{~cm} \mathrm{~s}^{-1}$. For our density profile at $t=1.1$ days we find a similar exponent but a very different proportionality constant: $\rho=$ $1.9 \times 10^{2} v^{-9.66} \mathrm{~g} \mathrm{~cm}^{-3}$. This difference can be the reason for the discrepancy in luminosity between analytic and numerical models. The difference in density may occur because the

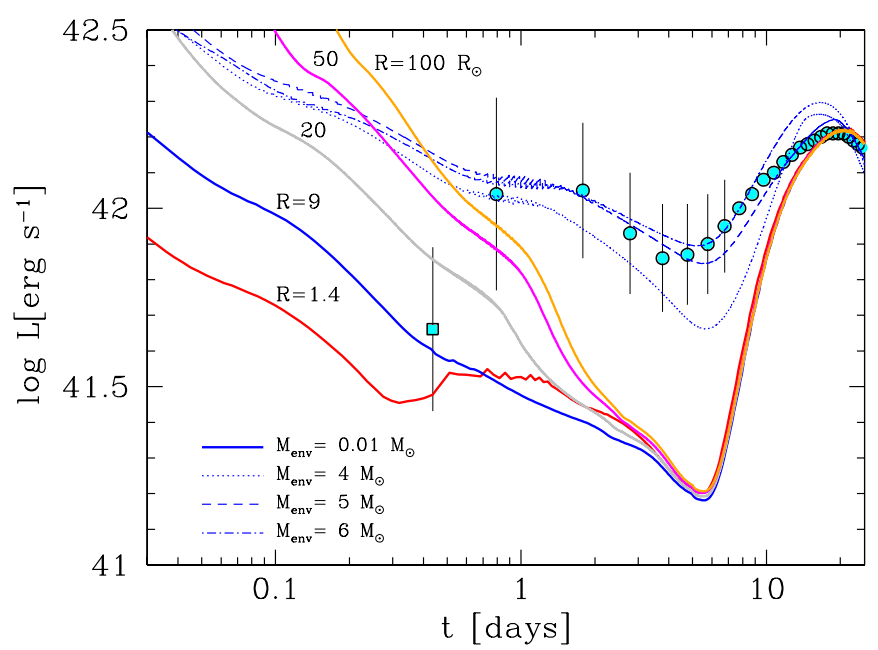

Figure 9. Bolometric LC for models with the same explosion energy as the optimal model, but different initial radii. Models whose variations in radius are accomplished by attaching essentially massless $\left(<0.01 M_{\odot}\right)$ envelopes to the He-rich layer of He8 model are shown by thick lines. Note that in this case larger radii produce higher early luminosity for $t<6$ days, although not high enough to explain the first peak of SN 2008D. Thin lines represent models with $9 R_{\odot}$ but with massive envelopes attached at different points of mass inside the He8 model, as indicated in the labels of the figure. These models, especially $M_{\mathrm{env}}=$ 5 and $6 M_{\odot}$, give a reasonable fit to the early observations with the exception of the earliest data point.

(A color version of this figure is available in the online journal.)

calibration of the post-explosion density profile used in CF08 was based on previous hydrodynamical calculations applied to a blue supergiant structure, useful to model SN 1987A, instead of a WR progenitor as required here.

\subsection{Different Progenitor Structure}

A larger value of the progenitor radius of $9 R_{\odot}$ was suggested by CF08 in order to explain the XRT of SN 2008D as completely thermal emission. On the other hand, Modjaz et al. (2009) found a similar radius by fitting the $\mathrm{CF} 08$ relations to the early data and assuming $E_{K}=2$ foe and $M_{\mathrm{ej}}=5 M_{\odot}$, as suggested by Soderberg et al. (2008). In Figure 7, we include the analytic models for these alternative physical parameters. In this case, the analytic models reproduce better the early luminosity with the exception of the earliest data point.

The improvement in the fit of the analytic models with larger radius suggests that adopting progenitors with large radii in our hydrodynamical simulations may allow us to reproduce the early data without resourcing to external ${ }^{56} \mathrm{Ni}$. We thus attached several envelopes in hydrostatic and thermodynamical equilibrium to the He-rich layer of our He8 model. The attached envelopes were integrated inward assuming a fixed stellar radius and varying the effective temperature and envelope mass so that the condition of continuity at the fitting point was achieved for mass, luminosity, pressure, and temperature. We also verified that the bottom of the envelope is cool enough to prevent nuclear reactions from developing in that region. This allowed us to generate a set of progenitors with radii of 9, 20, 50 and $100 R_{\odot}$.

The resulting bolometric LCs are shown with thick lines in Figure 9, where the other physical parameters are the same as previously adopted. From the figure it is clear that at $t \lesssim 5$ days models with larger radii produce slower cooling of the outer layers and higher luminosity, though not as large as the one predicted by the analytical models. After that time, all models look remarkably similar. Note that models start to converge at 


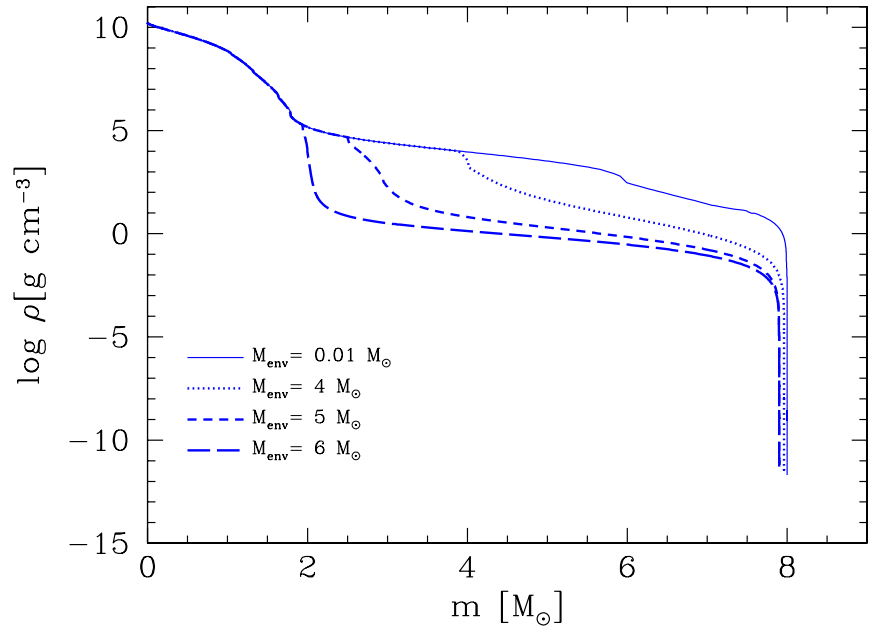

Figure 10. Initial density distributions as a function of mass for models with $9 R_{\odot}$ formed from model $\mathrm{He} 8$ but with massive envelopes attached at different points of mass, as indicated in the labels.

(A color version of this figure is available in the online journal.)

$t \approx 1.5$ days when the analytic expressions become invalid. Finally we see that even with a radius as large as $100 R_{\odot}$, we could not satisfactorily reproduce the early LC.

The analysis above was performed using nearly massless envelopes $\left(<0.01 M_{\odot}\right)$. The initial density profiles as a function of radius for these models are shown by thick lines in Figure 8. The shape of the density in the outermost layers does not follow the one used for the analytical models. This is reflected in a different, smoother, dependence of the luminosity on the progenitor radius than the prediction of the analytic models.

Alternatively, we calculated initial models with $9 R_{\odot}$ but with a substantial modification of the initial density distribution with respect to the He8 model. Specifically, we removed 4, 5 and $6 M_{\odot}$ of the original He8 model and attached new massive envelopes preserving the total mass $\left(8 M_{\odot}\right)$, out to a radius of $9 R_{\odot}$. These structures are shown in Figure 10 as a function of mass. The massless envelope model for $9 R_{\odot}$ is also included for comparison. Figure 9 shows the LCs resulting from these models (thin lines). The early observations can be reproduced reasonably well with these type of structures, excluding the earliest data point. In these cases, the shape of the density profile as a function of radius in the outermost layers (thin lines in Figure 8) are closer to the function assumed for the analytic models.

This means that, without considering the earliest data point, models with the larger radius and a modified density structure than those predicted by stellar evolution calculations can give a reasonable explanation of the early LC of SN 2008D as the cooling expansion of the outer envelope. However, the massiveenvelope models give a much poorer fit to the LC around the main peak, and they pose the additional problem of finding a physical explanation to justify a density profile that is different from those predicted by stellar evolution models. Note that some variations in the assumed pre-explosion density profile can be caused by effects of rotation, which were not included in our initial models. Nevertheless, the expected differences are much smaller than those needed to fit the early data of SN 2008D.

It is important note that recent binary stellar evolution calculation presented by Yoon et al. (2010) predicted a larger progenitor radius than that of $1.4 R_{\odot}$ obtained for our $\mathrm{He} 8$ model based on single stellar evolution. However, the radius comprising $95 \%$ of the mass is less than $1 R_{\odot}$, with the exception of the models that contain some hydrogen for which this radius can be as large as $5 R_{\odot}$ (see Table 2 of Yoon et al. 2010). For SN 2008D, the presence of a thin $\mathrm{H}$ envelope was firmly ruled out from spectroscopic analysis, e.g., T09 estimated a very low upper limit for the hydrogen mass fraction of $5 \times 10^{-4} M_{\odot}$. Therefore, the binary models are similar to our low-mass envelope models and are not consistent with the early observations of SN 2008D. This also becomes clear from the results presented by Dessart et al. (2011) based on the binary models of Yoon et al. (2010). All the LCs presented by Dessart et al. (2011) have a post-breakout luminosity that is much smaller than the one observed for SN 2008D, as noted by the authors and also according to our calculations.

\subsection{Binary Interaction Model}

As we noted previously, Dessart et al. (2011) also found that the observed luminosity of SN 2008D at early times was much larger than the one estimated by their models. Their calculations were done with a different hydrodynamical code and evolutionary initial models, as compared with our study. They considered it to be unlikely that light contamination from the host galaxy, or any light scattered by the CSM or pre-SN mass loss, could produce the large post-breakout luminosity. Moreover, CF08 estimated that the mass-loss rate of the progenitor was too low for the CSM to be optically thick. Alternatively, Dessart et al. (2011) suggested that the large observed luminosity could be due to the collision of the $\mathrm{SN}$ ejecta with a companion star in a binary system. However, they did not perform any detailed test of this scenario. Models that explain the early enhancement of the luminosity due to binary collision were proposed by Kasen (2010). These models depend on several parameters, such as binary separation, mass of the ejecta, shock velocity, and viewing angle. An analytic expression for the luminosity as a function of time for a viewing angle of $45^{\circ}$ is given in Equation (22) of Kasen (2010), with a stronger dependence on the shock velocity and the binary separation $(a)$ than on the ejected mass and the electron opacity. The shock velocity for SN 2008D can be estimated from the ejecta mass and explosion energy, assuming that $\kappa_{e^{-}}=0.2 \mathrm{~g} \mathrm{~cm}^{-3}$ for fully ionized electron-scattering of pure helium, and thus leaving $a$ as the only free parameter of the problem. Figure 7 shows an example of the binary collision model for a shock velocity derived using an explosion energy of 8.4 foe and an ejecta mass of $6.2 M_{\odot}$ as adopted in this paper. For this set of parameters the orbital separation required to reproduce the early luminosity is $a=1.8 R_{\odot}$. This value can be slightly increased to $a=2.8 R_{\odot}$ if we assume values of $E=3.7$ foe and $M_{\mathrm{ej}}=4.4 M_{\odot}$ appropriate for the He6 model (see Section 3.1). This very small constraint found for the binary separation may pose a problem for the binary collision scenario. Note that the typical binary separation of Galactic WR stars is $\gtrsim 10 R_{\odot}$, with very few exceptions (van der Hucht 2001). The situation might be remedied by assuming smaller viewing angles (see Figure 2 of Kasen 2010), but since we have no way to test this scenario, we will consider it a less likely possibility.

\section{CONCLUSIONS}

The early post-breakout emission of SN 2008D cannot be explained as the cooling of the outer layers of a normal WR star after the passage of the shock wave, as suggested in the literature. Alternatively, we have proposed a model that 
assumes a double-peaked ${ }^{56} \mathrm{Ni}$ distribution to explain this early emission as an attractive solution to this problem. Specifically, an amount of $0.01 M_{\odot}$ of ${ }^{56} \mathrm{Ni}$ was located in the outermost layers of the ejecta with $v \gtrsim 20,000 \mathrm{~km} \mathrm{~s}^{-1}$ _although the exact amount and distribution is subject to some uncertainties inherent to the data and the model itself. The assumption of external ${ }^{56} \mathrm{Ni}$ allowed us to reproduce very well both the early and late observations of SN 2008D. The presence of this high-velocity radioactive material may be caused by the formation of jets during the explosion. A multidimensional model would be required to confirm our suggestion in a self-consistent fashion.

Hydrodynamical models applied to WR stars predict a much larger contrast $(\gtrsim 0.9$ dex) between the initial dip and the peak of the LC due to heating by ${ }^{56} \mathrm{Ni}$ than what is observed for SN 2008D $(\approx 0.3$ dex $)$. Only with a substantial modification of the initial density structure that is predicted by stellar evolution calculations and for a larger progenitor radius, $9 R_{\odot}$, can the early emission be compatible with the cooling of the outer envelope. Even in this case the earliest observed point is not reproduced by the shock-cooling model and a much worse fit to the LC around the main peak is obtained. In addition, some physical explanation for such substantial changes in the initial density is needed to consider this as a possibility.

We also analyzed the possibility that the early emission was due to the interaction of the ejecta with a binary companion. This was based on the analytic predictions for the luminosity given by Kasen (2010). However, we found that the binary separation required to explain the early luminosity of SN 2008D is very small $\left(\lesssim 3 R_{\odot}\right)$, which poses a serious conflict for this interpretation.

Furthermore, we note that only the double-peaked ${ }^{56} \mathrm{Ni}$ model can reproduce the earliest observed data point as well as the rest of the LC. Both the binary interaction model and the modified density structure predict a larger luminosity at the earliest observed epoch.

Comparing our hydrodynamical models with the analytic models commonly adopted in the literature to reproduce the early emission (e.g., CF08 and RW11) we found: (1) the range of applicability of the analytic formula is very restricted. An upper limit of $\approx 1.5$ days after the explosion was found. In this range of time only two data points of SN 2008D are available. (2) The analytic models were calibrated with structures that may not apply to WR stars. This could be the reason for the very different value found for the proportionality constant of the relationship $\rho \propto v^{-n}$ for the post-explosion density profile as compared with the one used in CF08 (Equation (1)). (3) A modification of the radius of the star, as commonly applied for the analytic models, implies a different inner boundary condition for the density which cannot represent any stellar evolutionary solution. Therefore, conclusions based on these simple models should be taken with caution.

The analysis presented here shows the relevance of the early emission in our understanding of the progenitor structure previous to the explosion. We expect that current SN searches of increasing cadence will frequently detect more CCSNe in the early stages of their evolution. Then it will be possible to test whether the early behavior of SN 2008D is a peculiar to it or if it is a common feature of some type of SNe.

The authors gratefully acknowledge the helpful conversations with Keiichi Maeda and Gaston Folatelli. This research has been supported in part by the Grant-in-Aid for Scientific Research of MEXT (22012003 and 23105705) and JSPS (23540262) and by World Premier International Research Center Initiative, MEXT, Japan.

\section{REFERENCES}

Arnett, W. D. 1982, ApJ, 253, 785

Berger, E., \& Soderberg, A. M. 2008, GCN, 7159, 1

Bersten, M. C., Benvenuto, O., \& Hamuy, M. 2011, ApJ, 729, 61

Bersten, M. C., Benvenuto, O. G., Nomoto, K., et al. 2012, ApJ, 757, 31 Bietenholz, M. F., Soderberg, A. M., \& Bartel, N. 2009, ApJL, 694, L6 Blondin, S., Matheson, T., Modjaz, M., \& Berlind, P. 2008, CBET, 1205, 1

Campana, S., Mangano, V., Blustin, A. J., et al. 2006, Natur, 442, 1008 Chevalier, R. A., \& Fransson, C. 2008, ApJL, 683, L135

Clocchiatti, A., Wheeler, J. C., Brotherton, M. S., et al. 1996, ApJ, 462, 462

Deng, J., Tominaga, N., Mazzali, P. A., Maeda, K., \& Nomoto, K. 2005, ApJ, 624,898

Dessart, L., Hillier, D. J., Livne, E., et al. 2011, MNRAS, 414, 2985

Dessart, L., Hillier, D. J., Li, C., \& Woosley, S. 2012, MNRAS, 424, 2139

Drout, M. R., Soderberg, A. M., Gal-Yam, A., et al. 2012, ApJ, 753, 180

Ensman, L., \& Burrows, A. 1992, ApJ, 393, 742

Folatelli, G., Contreras, C., Phillips, M. M., et al. 2006, ApJ, 641, 1039

Gorosabel, J., de Ugarte Postigo, A., Castro-Tirado, A. J., et al. 2010, A\&A, 522, A14

Hammer, N. J., Janka, H.-T., \& Muller, E. 2010, ApJ, 714, 1371

Iglesias, C. A., \& Rogers, F. J. 1996, ApJ, 464, 943

Joggerst, C. C., Almgren, A., \& Woosley, S. E. 2010, ApJ, 723, 353

Kasen, D. 2010, ApJ, 708, 1025

Kong, E., \& Maccarone, T. J. 2008, ATel, 1355

Li, L.-X. 2008, MNRAS, 388, 603

MacFadyen, A. I., Woosley, S. E., \& Heger, A. 2001, ApJ, 550, 410

Maeder, A., \& Meynet, G. 1994, A\&A, 287, 803

Magee, N. H., Abdallah, J., Jr., Clark, R. E. H., et al. 1995, in ASP Conf. Ser. 78, Astrophysical Applications of Powerful New Databases, ed. S. J. Adelman \& W. L. Wiese (San Francisco, CA: ASP), 51

Malesani, D., Fynbo, J. P. U., Hjorth, J., et al. 2009, ApJL, 692, L84

Maund, J. R., Wheeler, J. C., Baade, D., et al. 2009, ApJ, 705, 1139

Mazzali, P. A., Valenti, S., Della Valle, M., et al. 2008, Sci, 321, 1185

Modjaz, M., Chornock, R., Foley, R. J., Filippenko, A. V., \& Li, W. 2008, CBET, 1222, A260000

Modjaz, M., Li, W., Butler, N., et al. 2009, ApJ, 702, 226

Nomoto, K., \& Hashimoto, M. 1988, PhR, 163, 1

Nomoto, K., Suzuki, T., Shigeyama, T., et al. 1993, Natur, 364, 507

Nomoto, K. I., Iwamoto, K., \& Suzuki, T. 1995, PhR, 256, 173

Owocki, S. P., Gayley, K. G., \& Shaviv, N. J. 2004, ApJ, 616, 525

Podsiadlowski, P., Hsu, J. J. L., Joss, P. C., \& Ross, R. R. 1993, Natur, 364, 509

Rabinak, I., \& Waxman, E. 2011, ApJ, 728, 63

Richardson, D., Branch, D., \& Baron, E. 2006, AJ, 131, 2233

Soderberg, A. M., Berger, E., Page, K. L., et al. 2008, Natur, 453, 469

Sugimoto, D., \& Nomoto, K. 1980, SSRv, 25, 155

Tanaka, M., Kawabata, K. S., Hattori, T., et al. 2012, ApJ, 754, 63

Tanaka, M., Tominaga, N., Nomoto, K., et al. 2009a, ApJ, 692, 1131 (T09)

Tanaka, M., Yamanaka, M., Maeda, K., et al. 2009b, ApJ, 700, 1680

Thompson, T. A., Chang, P., \& Quataert, E. 2004, ApJ, 611, 380

Tominaga, N., Tanaka, M., Nomoto, K., et al. 2005, ApJL, 633, L97

Valenti, S., Fugazza, D., Maiorano, E., et al. 2008, CBET, 1205, 2

van der Hucht, K. A. 2001, NewAR, 45, 135

Waxman, E., Mészáros, P., \& Campana, S. 2007, ApJ, 667, 351

$\mathrm{Xu}$, D., Watson, D., Fynbo, J., et al. 2008, 37th COSPAR Scientific Assembly, Montreal, Canada, 13-20 July 2008, 3512

Yoon, S.-C., Woosley, S. E., \& Langer, N. 2010, ApJ, 725, 940 